This document is the author's final manuscript of

M. Collet, M. Ouisse \& F. Tateo: Adaptive Metacomposites for Vibroacoustic Control Applications. IEEE Sensors Journal, 2014.

This paper was the cover of July 2014 issue.

This paper has been published by IEEE Sensors Journal and can be found at http://dx.doi.org/10.1109/JSEN.2014.2300052 


\title{
Adaptive Metacomposites for Vibroacoustic Control Applications
}

\author{
Manuel Collet, FEMTO-ST, CNRS, Besançon, France \\ and Morvan Ouisse, FEMTO-ST, ENSMM, Besançon, France \\ and Flaviano Tateo, FEMTO-ST, Besançon, France
}

June 2, 2014

\begin{abstract}
Research activities in smart materials and structures are very important today and represent a significant potential for technological innovation in mechanics and electronics. The growing interest of our society in the problem of sustainable development motivates a broad research effort for optimizing mechanical structures in order to obtain new functional properties such as noise reduction, comfort enhancement, durability, decreased ecologic impact, etc. In order to realize such a multi-objective design, new methods are now available which allow active transducers and their driving electronics to be directly integrated into otherwise passive structures. This new concept could allow fine control of the material physical behavior to induce new functional properties that do not exist in nature and that cannot be introduced by passive approaches. In this sense, we can speak of "integrated distributed adaptive metacomposites" that merges with the notion of programmable material. Through two different examples dealing with active acoustical impedance and elastodynamical interface, this paper present theoretical tools and validations for designing specific applications of this new technology.
\end{abstract}

\section{Introduction}

Constant research for developing new materials more adapted and more efficient for human needs, incorporating more and more new constraints such as the environmental damage (environmental impact, noise, recycling ...) led to study a new class of artificial multifunctional composite materials, namely the metamaterials. These new materials presenting specific physical properties due to their micro or nano structuring, were originally developed to synthesize new electromagnetic permittivity indices or negative permeability [1]. Recently, new research efforts have shown that the concepts could be transcribed in other areas of physics such as acoustics, mechanics or even robotics [2-4]. Thus, the realization of new structured materials has led to obtain very interesting new physical 
features which permit the design of integrated multi-functional structures capable of controlling their shape, vibrations [5] or distribution of acoustic and/or photonics energy $[1,6]$. Mainly based on achievement of periodic structures at different scales, the practical realizations are essentially passive and cannot be adapted in real-time. The recent technological revolution observed in the areas of Micro Electro Mechanical Systems can deeply extend the spectrum of future development of adaptive structures. One can now imagine full integration of hybrid systems consisting of adaptive materials, electronics, computing resources and power systems. The next generation of composite structures called 'smart metacomposite' should take full advantage of these technological advances for optimizing their behavior [7]. This new technology should lead to new relevant applications for noise and/or vibration control, for achieving integrated surface actuators or micro-acousto-optic devices for adaptive applications in telecommunications and health monitoring of structures.

The use of integrated and periodically distributed active (or hybrid) systems of transduction could allow fine control of the material physical behavior to induce new functional properties that do not exist in nature and that cannot be introduced by passive approaches. In this sense, we can speak of "integrated distributed adaptive metacomposites" conform to the notion of programmable material [8]. This approach represents a new challenge for creating intelligent hybrid materials and the associated distributed control strategy can solve inherent problems of classical active noise and vibration control strategies. Indeed, these intensively studied techniques have been proven to be efficient only on specific, well-known problems associated to very precise tuning of active systems because of the absence of controllability in general case, associated to lack of sufficient robustness for practical applications. They also require increase of complexity in terms of wiring, electronics, controllers and modifications of the baseline structure to successfully control structural or acoustic properties. Hence, among the problems to be considered, the broadband nature (from the low frequency -LFup to the high frequency -HF- and including the mid frequency -MF- range) of vibration and noise associated to composite structures and their assembly is still an open and challenging concern. This paper is a contribution to the challenges of designing, optimizing and manufacturing a new class of truly integrated and intrinsic smart metacomposites with ground breaking engineering performance in terms of mechanical and vibroacoustic behavior.

The concept of metacomposite has a specific meaning in this paper. This concept couples two different aspects in vibration and noise control:

- The first concept is connected to periodic structures theories usually connected to metamaterial concepts. In this field, intensive efforts were made in order to design and construct photonic or phononic crystals exhibiting wave band gaps that prevent energy to propagate in specific directions for particular frequencies; to create photonic/phononic crystals able to propagate light in anomalous and useful ways (i.e. negative refraction or artificial magnetism). This technique presents two main problems: the spatial modulation must be of the same order as the wavelength in the 
gap, and the position of the band gap cannot be easily changed since it strongly depends on the materials involved (Bragg's effect). A possible solution to these problems is found by using composites with locally resonant units: the periodicity of the crystal creates a stop band that can be shifted modifying the properties of the resonators. Liu et al. [9] have demonstrated that a resonant sonic crystal with blocks of rubber-coated lead balls exhibit a low-frequency sonic band gap, and the resonance can provide a maximum impedance mismatch to shield the airborne sound. The same effect can be obtained using Helmholtz resonators as showed by Fang et al. $[3,10]$ or $\mathrm{Hu}[11]$.

- The second concept is associated to vibration control through smart materials and implementation of specific strategies: shunted piezoelectric smart materials are employed for the metacomposite achievement by integrating into the metamaterial electronics and numerical components allowing implementation of adaptive and controlled behavior. In acoustics, implementation of optimal impedance can be used for controlling acoustical wave propagation [12]. We hence tend to extend the notion of programmable matter within the meaning of work presented in to vibroacoustic programming by showing that it is possible to implement specific partial derivative equations imitating a user-defined material dynamic equilibrium.

This paper aims at showing two examples of such new metacomposites for

controlling acoustic and mechanical power flows. In the first part, the concept of active acoustical skin is introduced and experimental validation is carried out. Innovative open field implementation is also presented to extend potential field of application for such a technology. The second part deals with smart mechanical interfaces for controlling absorption and transmission of elastodynamical energy. Plate interface prototype is also presented and characterized.

\section{Active Acoustic Skin : Metacomposite for Impedance Control}

\subsection{Introduction}

This part presents the acoustical capabilities of a network of distributed transducers connected with dedicated control strategy integrated as adaptive metacomposite for controlling acoustical impedance. The theoretical active skin can be depicted as a distributed interface in which a suitable control operator imposes the skin displacement $u(x, t)$ as a function of the fluid acoustic acceleration $\ddot{w}$ as presented in Fig. 1. This acceleration is directly proportional to the measured parietal acoustic pressure. The objective of the proposed strategy is to cancel positive group velocity of acoustic wave propagating in interaction with the metacomposite. Thus all incoming waves intercepting the smart liner only 


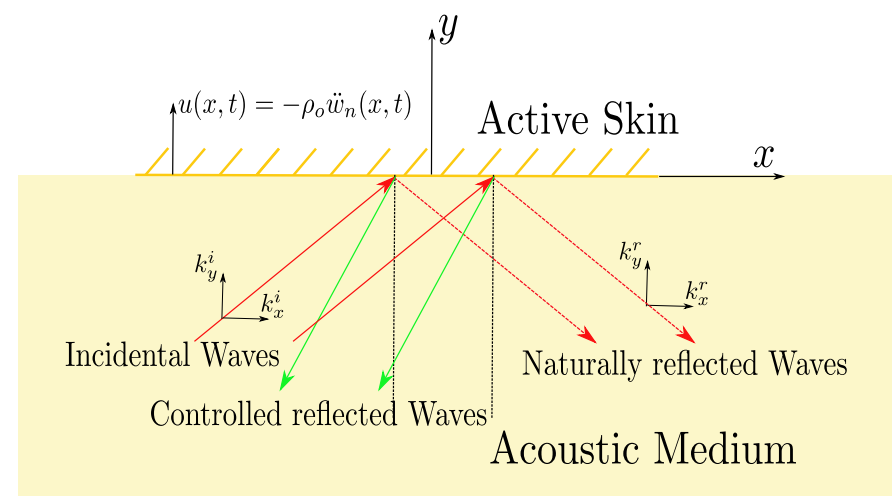

Figure 1: Acoustic waves interacting with the active surface

transport energy in the negative $(x)$ direction and becomes evanescent for the positive $(x)$ component of the wave number.

\subsection{Technological Integration of the acoustic adaptive meta- composite}

The control strategy based on partial differential equations (PDE) is obtained by using suitable mathematical tools and appears as an advection equation [7]. The theoretical implementation is validated for annihilating positive reflection of $2 \mathrm{D}$ acoustic waves. Its stability and robustness margins are also described in [13].

In order to experimentally implement the method, the control strategy is discretized as a first order time-space operator. The obtained quasicollocated architecture, composed of a large number of sensors and actuators, provides high robustness and stability. The proposed system is made of a periodically distributed set of active cells including one loudspeaker, one microphone, a DSP and conditioning electronics components as depicted in Fig. 2 [13]. The system is also implemented as an active liner in which all individual cells compute the local loudspeaker control signal by using pressure measurement coming from adjacent ones. Two identical control devices have been built, each composed of $4 \times 12$ cells, giving $(9 \times 36) \mathrm{cm}^{2}$ of active surface as shown in Fig. 2 .

\subsection{Experimental characterization}

The experimental set-up is composed of an acoustic wave guide with rectangular cross-section, audio amplifier with source loudspeaker on the input, two devices with active surfaces and non-reflecting acoustic termination on the output. A general view of the measurement device is presented in Fig. 3.

The experimental results are plotted in Fig. 4. The pressure transfer functions between pressure signals of microphones located on both sides of the ac- 

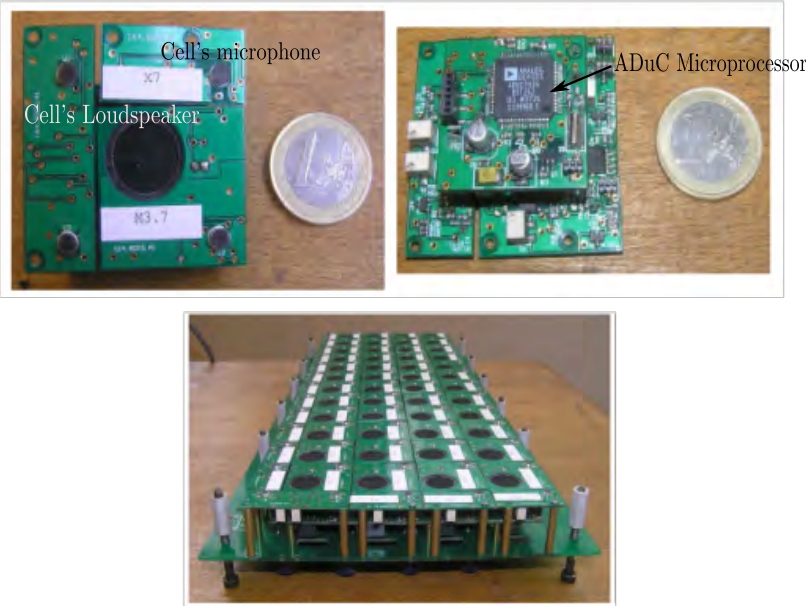

$4 \times 12$ cells active skin

Figure 2: Individual active acoustic cells and general view of the realized liner made of $4 \times 12$ cells: elementary cell (1 loudspeaker with 4 adjacent microphones); associated DSP; $4 \times 12$ cells active skin

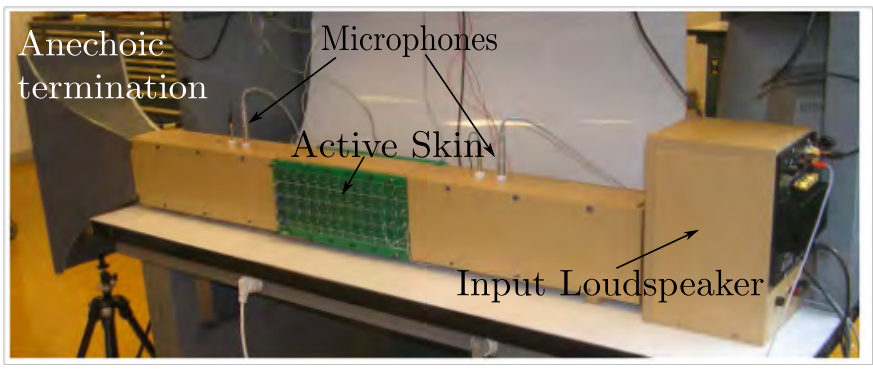

Figure 3: Acoustic waves interacting with the active surface

tive acoustical skin with and without control shows how the active liner made of metacomposite can substantially modify sound reflectivity of the acoustical interface and reduce the propagation of acoustic waves outside the duct. The induced transmissibility is decreased of about $30 \mathrm{~dB}$ at the maximum efficiency. The system is efficient on a wide frequency band, namely between $800 \mathrm{~Hz}$ and $1.5 \mathrm{kHz}$. The lower cutting frequency is induced by the loudspeaker dynamic while the second comes from the computational time of cell's DSP for computing the distributed command. 


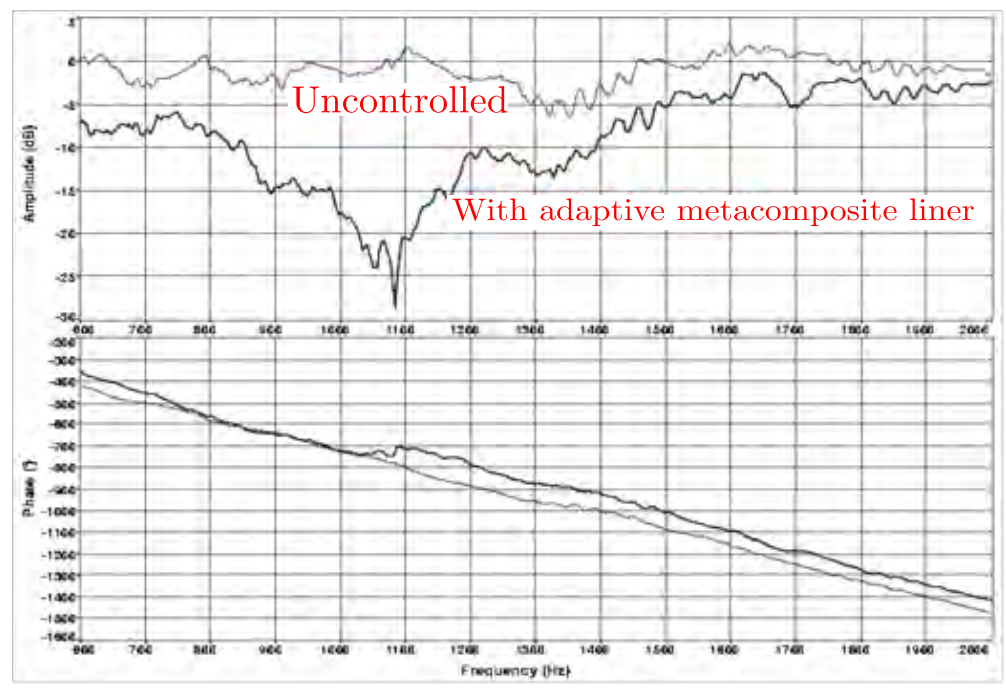

Figure 4: Transfer function between the input and output microphone, active surfaces with and without control.

\subsection{Potential for controlling open field acoustic}

The optimal generalized impedance used to control the simple acoustic duct problem previously presented can also be implemented in more complex systems. We consider here an open acoustic field problem constituted of a $2 D$ domain bounded on the external half-circle by a classic Sommerfeld condition and rigid on the bottom boundaries as shown on the top of figure 5. This system is excited by a pressure point source normalized to $1 . e^{+3} P a$ and equipped by active skins symmetrically located between $0.1 \mathrm{~m}$ to $0.4 \mathrm{~m}$ from the origin. The theoretical partial derivative equation is implemented to simulate the active impedance as described in [7]. The advection velocity is here chosen equal to the acoustic wave velocity $c_{o}=340 \mathrm{~m} . \mathrm{s}-1$.

Both figures in the bottom part of figure 5 show the controlled and uncontrolled norm of the pressure field for an excitation at $440 \mathrm{~Hz}$. We immediately observe that the pressure field distribution is strongly modified by the active skin. The acoustic energy is constrained to focus on just above the source and floor pressure near the bottom boundary is amply decreased. The active generalized impedance is also able to produce quiet sub-domain.

To extend these results obtained for a particular frequency, we present on figure 6.a the difference of controlled and uncontrolled ratio of radiated external acoustic power flow normalized by the input one, passing through the Sommerfeld boundary condition. The figure radius correspond to the computed frequencies and the angles to the position onto the external half circle boundary condition. On figure 6.b is plotted the percentage of the total emitted acoustical power flow when the active liners are on duty. We immediately observe 


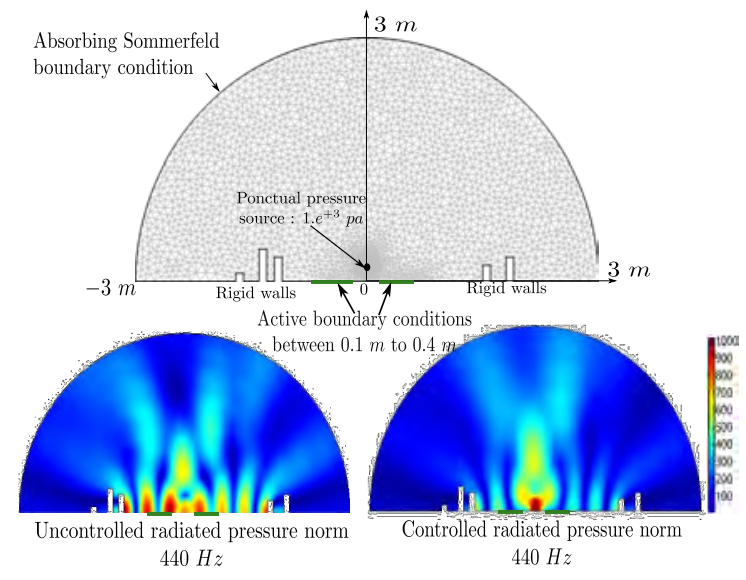

Figure 5: Open field treatment. On the top : system description; bottom left : norm of the pressure field without control, bottom right : norm of the pressure field with control.

that the two active boundaries conditions located on the bottom part of the domain are sufficienty efficiency to greatly modify the directionality of sound power flow. As depicted on figure 6.a we observe at low frequency, before 670 $\mathrm{Hz}$, an attenuation of grazing flow by more than $50 \%$. After $650 \mathrm{~Hz}$ we note a strong decrease of noise power emitted at $45^{\circ}$ and also but less at about $80^{\circ}$. We can underline an increase of the radiated power flow for some frequencies under certain directions. The global sound intensity presented in figure 6.b is always decreased by the active liners, by more than $55 \%$ at $600 \mathrm{~Hz}$ and $10 \%$ at the higher frequencies.

Through all these results, we underline here the potential of such distributed strategy to be used in complex acoustic system (even non controllable) to control noise distribution. The efficiency depends on the targeted wave length but also on the length of the equipped interface.

\subsection{Conclusion}

In this section, we present a so-called adaptive acoustic liner made of a distribution of individual cells integrating micro-controller, sensor and actuator, power supply, signal amplification, signal conditioning to control acoustical impedance. The metacomposite has a great merit of being very effective and allows an important attenuation of the acoustic transfer without the necessity of very strong displacements of the membrane interfaces [13]. The last section of this first part shows up the potential of this strategy for modifying noise distribution 


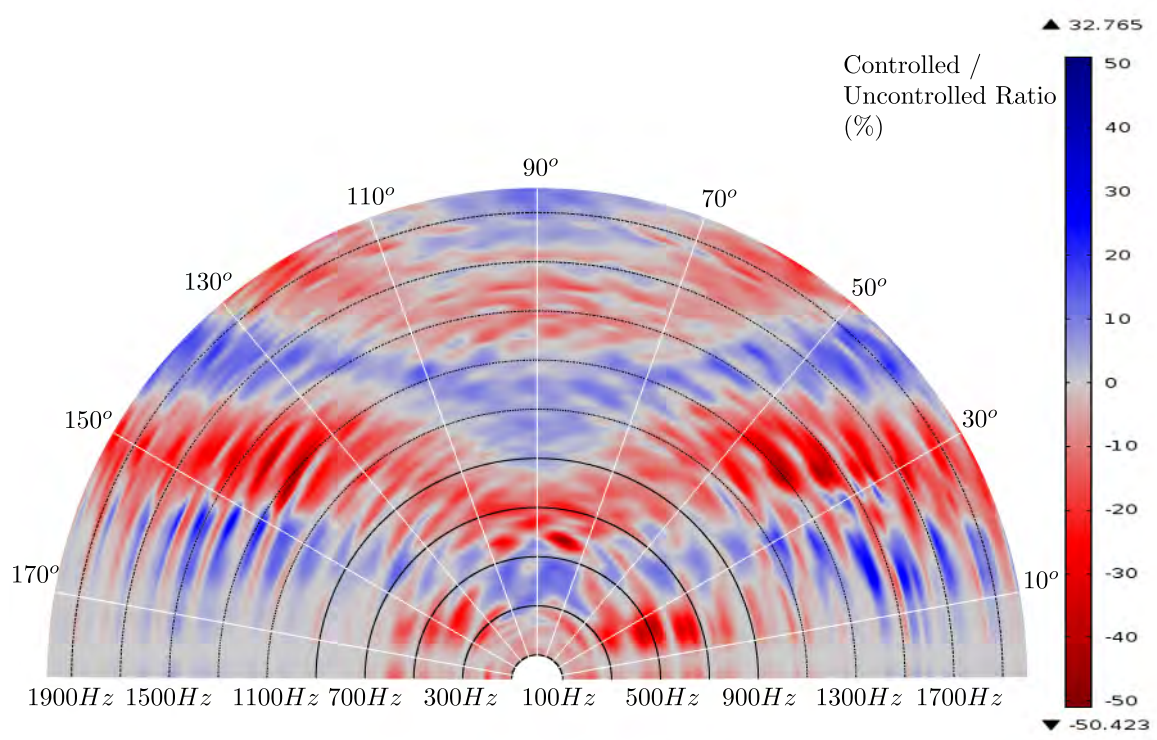

(a) Normalized radiated power flow

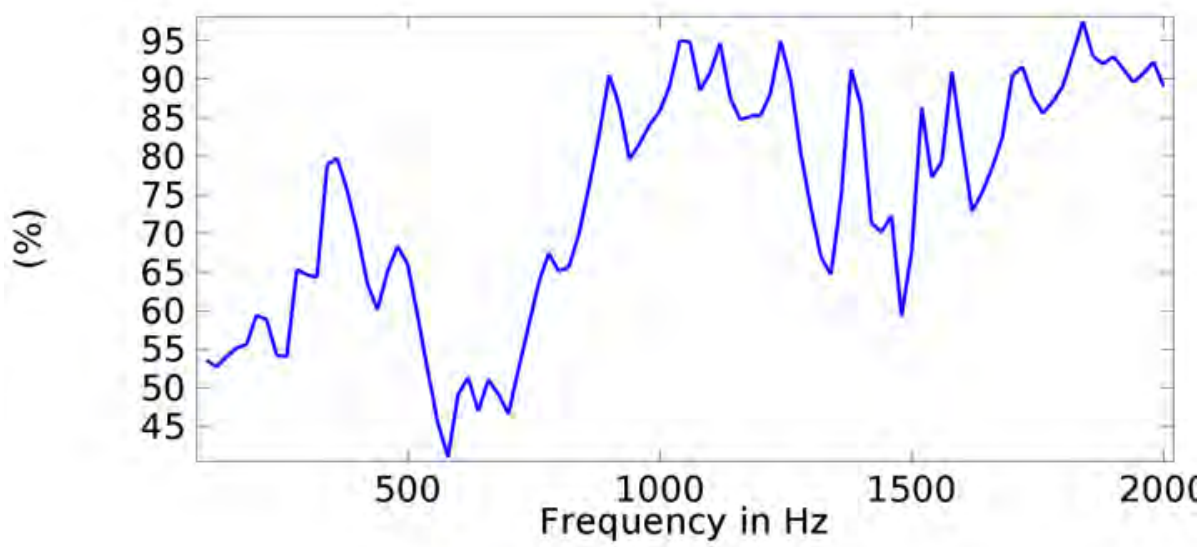

(b) Attenuation in radiated power flow

Figure 6: a/ Ratio of controlled and uncontrolled radiated external acoustic power flow passing through the Sommerfeld boundary condition normalized by the emitted source power. $\mathrm{b} / \%$ of the uncontrolled total acoustical power flow emitted in far field when the active interfaces are on

in open field problems and its capacity to protect specific zone from acoustic sources. Potential applications of acoustic impedance control by metacomposite 
adaptive skins are also numerous but dedicated technological solutions should be developed to obtain fully adaptive and integrate implementations.

\section{Smart mechanical metacomposite: how to con- trol absorption and transmission of elastody- namical energy}

\subsection{Introduction}

The concept of metacomposite is adapted here to the control of energy flow in 2 D elastodynamical wave guides by using shunted piezoelectric patches. A classical approach is to design adaptive metacomposite to create local resonances by coupling piezoelectric patches to a resonant external circuit as illustrated in [5]. This concept involves a periodic array of simple RL-shunted piezoelectric patches mounted on the structure to passively control the propagation of elastic waves and the subsequent vibration field. Periodically induced impedancemismatch zones generate broad stop bands, i.e., frequency bands where waves are attenuated. The tunable characteristics of shunted piezo-patches allow the equivalent mechanical impedance of the structure to be tuned so that stop bands are generated over desired frequency ranges. The presence of a resistance in the shunt circuit generates a damped resonance of the electrical network that allows the energy dissipation mechanism of shunted piezo-patches to be exploited to dampen the amplitude of vibration also outside the stop bands. The original periodic shunting concept was numerically demonstrated on rods and fluid-loaded axisymmetric shells in [14]. More recently, this strategy was extended to flat plates [15-17], where Bloch theorem was used to predict the dispersion properties of the resulting periodic assembly.

The metacomposite studied in this part is made of periodically shunted piezoelectric patches on different optimal electric shunt circuits designed by using methodologies developed in [18]. An equivalent approach is available for acoustic coupling in [19]. The optimal electric impedances appear to be a quasiconstant resistance in series with negative capacitance in all situations. This particular metacomposite is not based on the same physical principles as distributed resonant circuits but appears as the optimal solution for controlling energy velocity or wave absorption property. Experimental validation of this approach has been made on beams [20] and fully covered plate characterization in [15]. The main point of this paper concerns the plate implementation of a distributed interface made of shunted $R C_{n e g}$ piezoelectric metacomposite separating two parts of the supporting plate. The experimental characterizations also deal with the metacomposite behavior but also its effect on the energy balance inside the plates system and a complete analysis of power flow interacting with the smart interface. Different circuit parameters are also implemented to observe different behaviors on the energy diffusion through the smart metacomposite interface. 


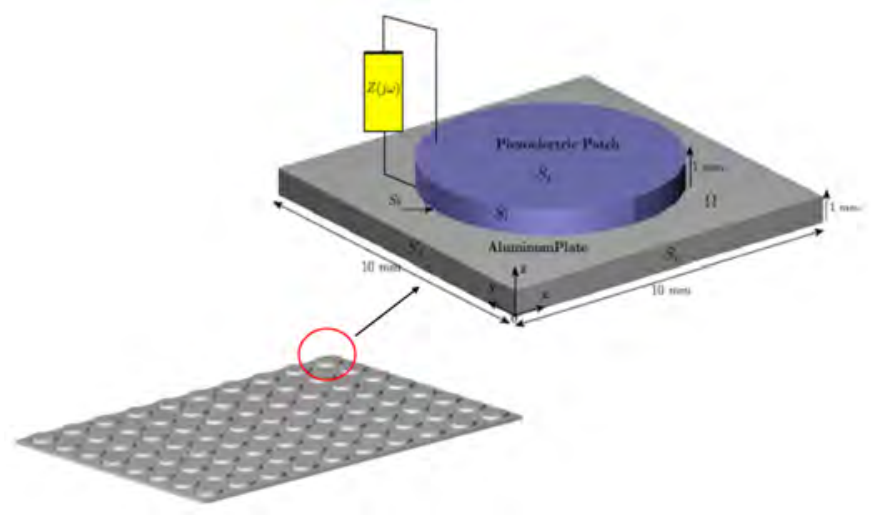

Figure 7: Metacomposite made of piezocomposite cells

\subsection{The piezoelectric metacomposite}

The considered metacomposite is made of piezocomposite cells connected to specific electrical impedance (Fig. 7). The supporting plate material is standard aluminum with $0.1 \%$ of hysteretic damping ratio and the piezoelectric material is PZT 2.

The dedicated numerical tools [18] allow the optimization of the piezoelectric shunt impedance $Z(i \omega)$. The first criterion is used to enhance the metacomposite capability for decreasing structural energy transmission. It is based on the minimization of the flexural wave energy velocities [18]. The second criterion improves wave's absorption. It is based on the computation of the dissipated electrical energy into the electrical shunt [18].

\subsection{Energy propagation with optimal impedance}

Dispersion curves obtained with open shunt circuit and optimal impedances controlling transmission and absorption are plotted in Fig. 8. We observe a large modification of $A 0$ mode when transmission is controlled on the left part of Fig. 8. The corresponding energy velocity is also largely decreased and such metacomposite appears as an energy barrier for flow carried by mechanical bending movement. Absorption optimization does not lead to the same behavior. We do not observe energy transmission cancellation (dispersion curves of propagation modes are not modified) but only a strong increase of the waves decay rates (i.e. the ratio between real and imaginary parts of the complex wave numbers).

The corresponding optimal impedances are plotted in Fig. 9. We immediately observe that both criteria lead to connect quasi constant negative capacitance for adjusting the reactive part of the electrical impedance to the mechanical one. When transmission is targeted, the optimal resistance (the active part) is negative in order to control the natural dissipative term added into the model and lead the system to a fully conservative one. For absorption, the resistance 


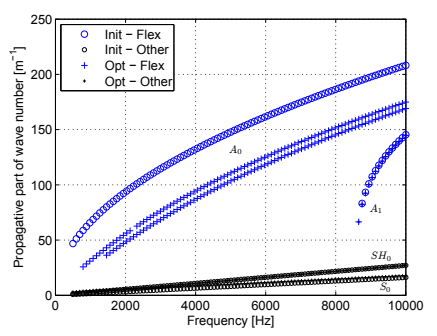

Transmission

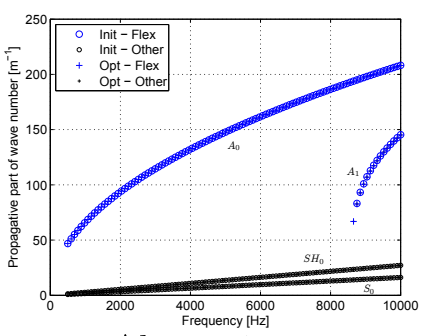

Absorption

Figure 8: Propagation parts of the wave number $k_{x}$ along $(O x)$, (only propagating waves included). o: initial value of shunt (arbitrary); + : optimal value of shunt. Large marks correspond to flexural waves, small marks correspond to other waves.

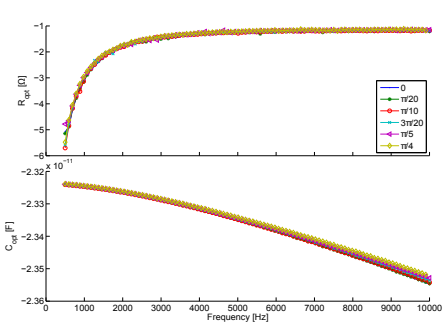

Transmission

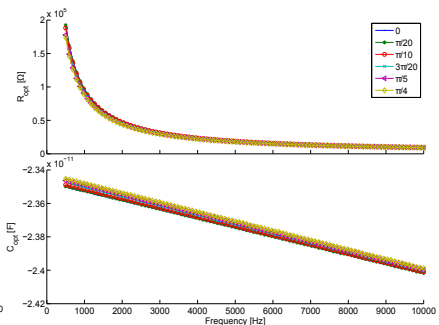

Absorption

Figure 9: Optimal electric impedance, represented as equivalent resistances and capacitances, obtained along 6 direction forming an angle with $(O x)$ axis of $n \pi / 10$ with $n$ being all integers from 0 to 5 .

is positive to damped waves into the periodic metacomposite. For both cases, the optimal impedance is almost the same whatever the wave incident angle is, which guarantees robustness and efficiency of the concept.

\subsection{Validation on a periodically semi-distributed set of adaptive cells}

Up to that point, the optimizations of the smart metacomposite which have been proposed were performed by considering infinite periodic structures. The objective of this section is to illustrate the applicability of the designs for integration into real-life finite structures. The optimal impedance is then applied on a finite set of shunted piezocomposite cells distributed on a part of a totally free plate system submitted to a point force in one corner, as described in figure 10. The harmonic response of this system is then computed on the frequency band of interest when optimal impedances, obtained by criteria $\mathrm{Crit}_{1}$ (minimization of transmission) or $\mathrm{Crit}_{2}$ (maximization of dissipation), are connected or not to 


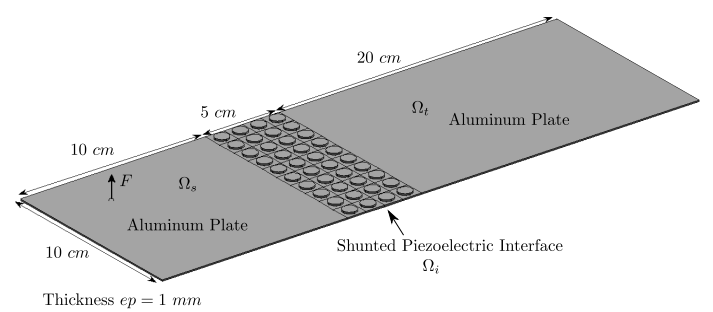

Figure 10: 3D piezocomposite periodic semi-distributed cells as plate interface

each patch. The figure 11 shows the corresponding velocity root mean square distribution for uncontrolled plate and both types of optimal impedances.

These numerical results clearly show a strong influence of the modifications of the wave dispersion characteristics on the standing wave responses plotted in figure 11 especially when $\mathrm{Crit}_{1}$ is used. In particular, at $2550 \mathrm{~Hz}$, a large attenuation of the dynamic response of the system can be observed when optimal shunts are connected. In this case, when the shunts are optimized for canceling mechanical energy flow $\left(\right.$ Crit $\left._{1}\right)$, the location of the nodal line are also modified. With an optimization aiming at damping energy in the electric resistance $\left(\mathrm{Crit}_{2}\right)$, only to a standard attenuation can be observed. At $8000 \mathrm{~Hz}$ when Crit $_{1}$ optimal shunts are connected, a large part of the system energy remains in its left part where the applied force is located. The smart metacomposite interface also changes the system admittance and filter wave diffusion by increasing its reflexibility properties. The energy diffusion is also clearly condensed into the left part of the system with a largely decreased amplitude compared to the one obtained with open circuit and $\mathrm{Crit}_{2}$ optimal shunt. With this type of shunt something similar to a wave trap effect can be observed. With $\mathrm{Crit}_{2}$ optimal shunt, an improvement of the damping effect leading to vibration attenuation can be observed, without wave trap behavior.

\subsection{Experimental Validation : a plate metacomposite in- terface}

\subsubsection{The system design}

The basic structure of the metacomposite comprises an aluminum plate and array of 75 piezoelectric patches as depicted in Figure 12. The geometry and material properties for the plate and piezoelectric materials are listed in Table 1. The plate is suspended from a rigid frame by metallic wires in order to approximate free-free boundary conditions. The plate metacomposite interface is equipped with 75 piezoelectric patches (Ferroperm Industries $P Z 26$ series) arranged in a regular $15 \times 5$ array as seen in Figure 12. The dimension of the piezoelectric patches are listed in Table 1. 
Table 1: Geometry and physical properties of the system.

\begin{tabular}{|c|c|c|c|}
\hline \multirow[t]{6}{*}{ Plate } & Length & 2100 & $\mathrm{~mm}$ \\
\hline & Height & 1050 & $\mathrm{~mm}$ \\
\hline & \multicolumn{2}{|c|}{ Thickness3 } & $\mathrm{mm}$ \\
\hline & $\begin{array}{l}\text { Mass } \\
\text { den- } \\
\text { sity }\end{array}$ & 2700 & $\mathrm{~kg} / \mathrm{m}^{3}$ \\
\hline & $\begin{array}{l}\text { Young's } \\
\text { modu- } \\
\text { lus }\end{array}$ & $70 \cdot 10^{9}$ & $N / m^{2}$ \\
\hline & $\begin{array}{l}\text { Poisson's } \\
\text { ratio }\end{array}$ & 0.33 & - \\
\hline \multirow[t]{7}{*}{ Piezo } & Length & 50 & $\mathrm{~mm}$ \\
\hline & Height & 50 & $\mathrm{~mm}$ \\
\hline & \multicolumn{2}{|c|}{ Thickness0.5 } & $\mathrm{mm}$ \\
\hline & $\begin{array}{l}\text { Mass } \\
\text { den- } \\
\text { sity }\end{array}$ & 7650 & $\mathrm{~kg} / \mathrm{m}^{3}$ \\
\hline & $\begin{array}{l}\text { Poisson's } \\
\text { ratio }\end{array}$ & 0.31 & - \\
\hline & $\begin{array}{l}\text { Dielectic } \\
\text { loss }\end{array}$ & $<0.05$ & $\%$ \\
\hline & $\begin{array}{l}\text { Coupling } \\
\text { factor } \\
\left(k_{31}\right)\end{array}$ & 0.33 & - \\
\hline
\end{tabular}



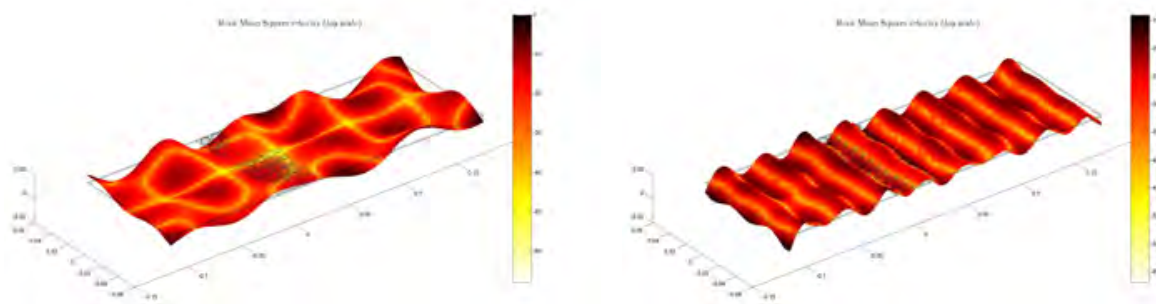

(a) Open circuit patches
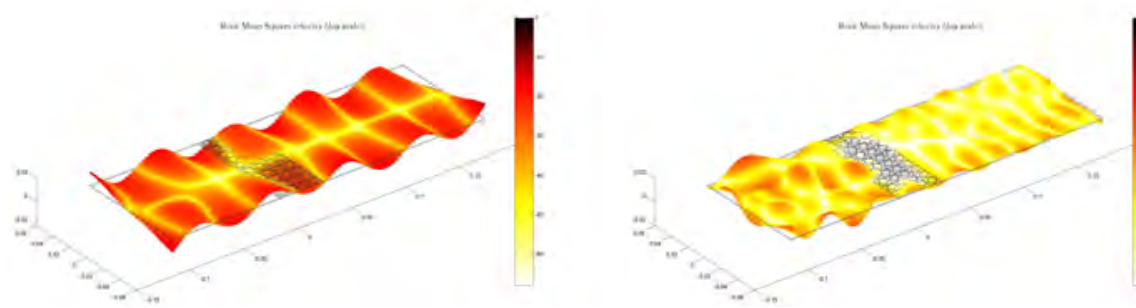

(b) Crit $_{1}$ Optimal Shunt
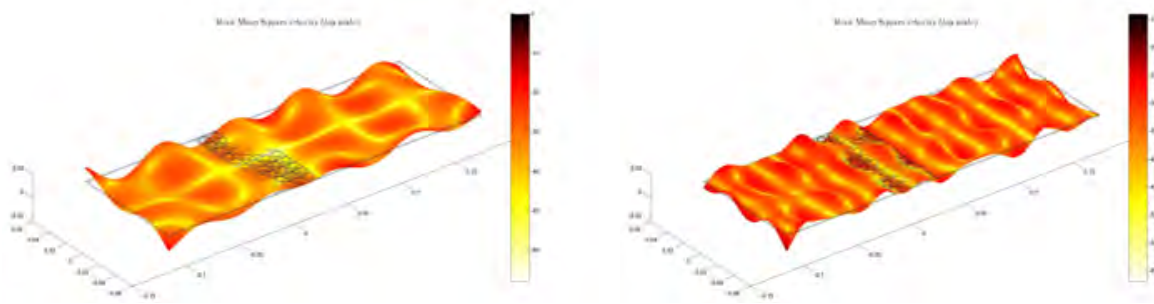

(c) Crit $_{2}$ Optimal Shunt

Figure 11: Velocity root mean square level of the system submitted to an external point force at $2550 \mathrm{~Hz}$ (left) and $8000 \mathrm{~Hz}$ (right) for all piezoelectric cells in open circuit (a), connected to optimal shunt impedance obtained with Crit $_{1}$ (b) and $\mathrm{Crit}_{2}$ (c)

The patches and the depth of the patch array were selected based on consideration of their size relative to the shortest structural wavelength of interest. The array was intended to be effective up to a frequency of $5000 \mathrm{~Hz}$. The plate's dispersion relation for the $A_{0}$ flexural mode at $5000 \mathrm{~Hz}$ has a wavelength of 30 $\mathrm{cm}$. With patches having dimensions of $5 \times 5 \mathrm{~cm}$, there will be no charge cancellation on an individual patch as would occur if the patch dimensions were comparable to a wavelength. Further, the depth of the patch array is $35 \mathrm{~cm}$ such that it spans more than a wavelength at $5000 \mathrm{~Hz}$. Since the array elements are uncoupled electronically, each element within the array would still function at the highest frequency of interest, even though the entire array depth would be comparable to a wavelength. The thickness of the patch was selected considering the constraints of the electric circuit and the nature of the control technique 


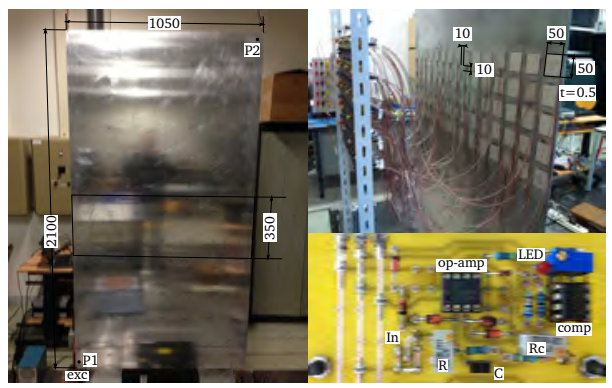

Figure 12: Experimental rig. Left aluminum plate dimension and input applied force at point $P_{1}$, single-point response measurement at $P_{2}$. Top right actuator's lattice. Bottom right actual electronic circuit. All dimensions are expressed in $\mathrm{mm}$.

as proposed by [20] to work with sufficiently high capacitance while maintaining a good effective piezoelectric coupling a thickness of $0.5 \mathrm{~mm}$ was selected.

Each actuator is connected to an independent analog circuit reproducing the negative capacitance effect in serial with a resistance. The circuit can be ruled in order to reproduce both comprise given by optimizing reflectivity $\left(\mathrm{Crit}_{1}\right)$ leading to use low input resistance of $40 \Omega$ and optimizing absorption with a high input resistance of $400 \Omega\left(\right.$ Crit $\left._{2}\right)$. On the bottom corner of figure 12 is depicted the actual circuit used during the experiment. This circuit contains some passive components such as the resistances $R, R_{c}$ and the capacitance $C$. However the targeted circuit behavior can only be achieved introducing an active component, namely an operational amplifier. This control technique requires a tuning of the synthetic capacitance around the capacitance value of the piezoelectric ceramic, this value is intrinsic and depends upon the material properties and the geometry. This particular point strongly affects the circuit's behavior, during operation a voltage overload may be observed (op-amp saturation) [20]. In order to mitigate this effect the circuit's layout proposed by Beck were modified adding a complementary circuit able to detect this specific instability. Basically a comparator circuit were introduced in correspondence of the op-amp output in order to identify these voltage overloads and to allow experimental circuit tuning.

\subsubsection{Results and discussions}

In figure 13 is depicted the plate's frequency response functions measured at point $P_{1}$ (near the point source) on the left and at point $P_{2}$ (upstream of active interface) on the right. The mobility function is measured over the frequency range $500-4000 \mathrm{~Hz}$. In the low frequency band the system cannot be very effective, however, beyond $1000 \mathrm{~Hz}$ the control system is activated, causing an appreciable vibration reduction up to $4 \mathrm{kHz}$ especially at point $P_{2}$. Analyzing the two lines representing the uncontrolled system in blue line and the controlled 


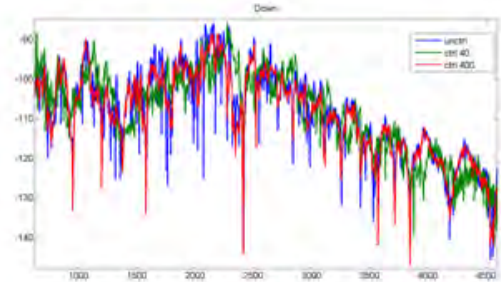

(a) $P_{1}$ mobility function

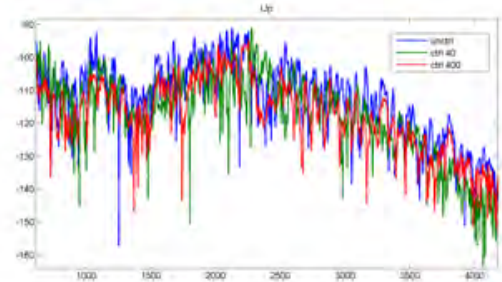

(b) $P_{2}$ mobility function

Figure 13: FRF plot comparison between the uncontrolled system (blue line) and the controlled system with reflective metacomposite (green line) and absorbing metacomposite (red line). (a) $P_{1}$ measurement, (b) $P_{2}$ measurement.

system with reflective metacomposite $\left(\mathrm{Crit}_{1}\right)$ in green line and absorbing metacomposite $\left(\mathrm{Crit}_{2}\right)$ in red line, we observe a small reduction of the system's response at point $P_{1}$ but some strong modifications on the locations of modal peaks and a large decrease of vibration amplitude at point $P_{2}$. The control of the dynamical behavior of the plate especially the movements observed on the resonance frequencies depict a large modification of the sub-domain responses downstream and upstream the active metacomposite interface.

The modification of the plate's internal dynamics within each of the regions of the plate (upstream of the active interface, within the active interface, and downstream of the active interface) can be observed through the time-averaged kinetic energy distribution. A clear depiction of the impact of the active interface may be obtained by considering the area distribution of kinetic energy (essentially plotting the square of the local velocity on the plate) as depicted in Figure 14. The top row of plots depict the kinetic energy distribution among the three control configurations at $2500 \mathrm{~Hz}$. On the left, (control OFF) the kinetic energy is evenly spread over the entire plate. In the middle, the system's response is slightly modified by the low-damping, low resistance of $40 \Omega$ $\left(\mathrm{Crit}_{1}\right)$. In contrast, as a result of increasing the value of the resistance of $400 \Omega$ $\left(\mathrm{Crit}_{2}\right)$, there by increasing the damping, the kinetic energy distribution of the plate is clearly modified; the response is most strongly confined to the portion of the plate below the active interface. Considering a higher frequency example, for instance $3000 \mathrm{~Hz}$ as depicted in the lower plots of Figure 14, the behavior of the system is radically modified. For the lightly-damped control case 1, the vibratory energy of the plate is even more noticeably confined in the upstream subdomain $\Omega_{1}$ as compared to the response at $2500 \mathrm{~Hz}$. In fact, the vibration amplitude is increased in the upstream region for case 1 as compared to the control off case. For the more heavily-damped control of case 2, the reflective behavior is no longer present, and the driving control mechanism is a dominantly dissipative effect that makes the kinetic energy field weaker everywhere through suppression of a reactive vibratory response. 


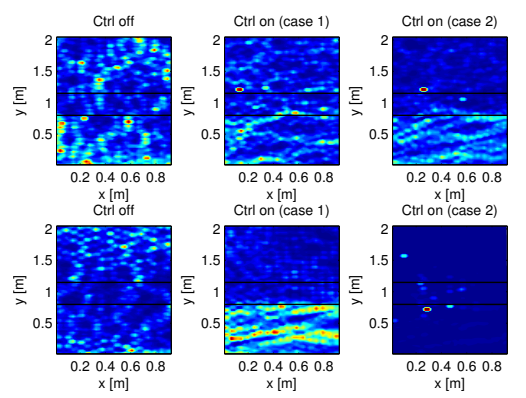

Figure 14: Plate kinetic energy field: (left) control OFF, (center) control ON Crit $_{1}$, (right) control ON Crit 2 . (Top) $2500 \mathrm{~Hz}$, (bottom) $3000 \mathrm{~Hz}$. Horizontal black lines indicate location of active interface.

\subsubsection{Conclusions}

The part clearly underlines the potential of innovation that stands on the use of active metacomposite for controlling vibrationnal power flow. Specific methodological tools, beam and plate demonstrators have been developed to design and characterize such new composite materials. These preliminary results are encouraging, however further analysis will be required in order to thoroughly understand the electro-mechanical coupling related to this control technique in order to improve technical integration and solutions for energy management.

\section{Conclusion}

In this paper, we present designs and characterizations of two applications of integrated metacomposites made of periodically arranged adaptive cells for controlling vibroacoustique power flow in acoustic or mechanical systems. This study provides methodologies to optimize electromechanical behavior of two metacomposites for 2-3 D wave guides applications. The main purpose of this work was first to present the dedicated numerical approaches suitable to compute and optimize the multi-modal wave dispersions or interface impedance. Experimental applications highlight the real potentialities of such technology for controlling complex vibroacoustic interactions by demonstrating its robustness and efficiency.

Such concept can be extended to different functionalities like shape control or new generation of smart and integrated Structural Health Monitoring systems that can be used for prognostics. Even if large project are devoted to develop such technologies we are still far away from real material-integrated sensing systems but the presented results encourage us to continue on this way. Use of MEMS technologies can offer a suitable way to obtain more integrated systems. 


\section{Acknowledgment}

The authors would like to thank the French National Agency projects under grant number NT09-617542 and Labex Action (ANR-11-LABX-01-01) for their support.

\section{References}

[1] V. G. Veselago, "Electrodynamics of substances with simultaneously negative value of sigma and mu," Sov Phys Uspekhi-USSR, vol. 10, p. 509, 1968.

[2] S. Yang, J. Page, Z. Liu, M. Cowan, C. Chan, and P. Sheng, "Ultrasound tunneling through 3D phononic crystals," Physical Review Letters, vol. 88, no. 10, p. 104301, 2002.

[3] N. Fang, D. Xi, J. Xu, M. Ambati, W. Srituravanich, C. Sun, and $\mathrm{X}$. Zhang, "Ultrasonic metamaterials with negative modulus," Nature $\mathrm{Ma}$ terials, vol. 5, no. 6, pp. 452-456, 2006.

[4] T. Wu, T. Wu, and J. Hsu, "Waveguiding and frequency selection of Lamb waves in plate with periodic stubbed surface," Physical Review B, vol. 79, p. 104306, 2009.

[5] O. Thorp, M. Ruzzene, and A. Baz, "Attenuation and localization of wave propagation in rods with periodic shunted piezoelectric patches," Smart Materials and Structures, vol. 10, no. 5, p. 979, 2001.

[6] F. Bongard, H. Lissek, and J. . Mosig, "Acoustic transmission line metamaterial with negative/zero/positive refractive index," Physical Review B, vol. 82 , no. 9 , p. $094306,2010$.

[7] M. Collet, P. David, and M. Berthillier, "Active acoustical impedance using distributed electrodynamical transducers," Journal of Acoustical Society of America, vol. 125, no. 2, p. 882894, 2009.

[8] T. Toffoli and N. Margolus, "Programmable matter: Concepts and realization," Physica D: Nonlinear Phenomena, vol. 47, no. 1-2, pp. 263 - 272, 1991.

[9] Z. Liu, X. Zhang, Y. Mao, Y. Zhu, Z. Yang, C. Chan, and P. Sheng, "Locally resonant sonic materials," Science, vol. 289, no. 5485, pp. 1734-1736, 2000.

[10] M. Ambati, N. Fang, C. Sun, and X. Zhang, "Surface resonant states and superlensing in acoustic metamaterials," Physical Review B, vol. 75, no. 19, p. $195447,2007$.

[11] X. Hu, C. Chan, and J. Zi, "Two-dimensional sonic crystals with helmholtz resonators," Physical Review E, vol. 71, no. 5, p. 055601, 2005. 
[12] B. Betgen, M. Galland, and E. Piot, "Implementation and non-intrusive characterization of a hybrid active-passive liner with grazing flow," Applied Acoustics, vol. 73, p. 624628, 2012.

[13] P. David, M. Collet, and J. M. Cote, "Experimental implementation of acoustic impedance control by 2 d network of distributed smart cells," Smart Materials and Structures, vol. 19, no. 3, p. 035028, 2010.

[14] O. Thorp, M. Ruzzene, and A. Baz, "Attenuation of wave propagation in fluid-loaded shells with periodic shunted piezoelectric rings," Smart Materials and Structures, vol. 14, no. 4, p. 594, 2005.

[15] F. Casadei, M. Ruzzene, L. Dozio, and K. Cunefare, "Broadband vibration control through periodic arrays of resonant shunts: experimental investigation on plates," Smart Materials and Structures, vol. 19, no. 1, p. 015002, 2010 .

[16] A. Spadoni, M. Ruzzene, and K. Cunefare, "Vibration and wave propagation control of plates with periodic arrays of shunted piezoelectric patches," Journal of Intelligent Material Systems and Structures, vol. 20, no. 8, pp. 979-990, 2009.

[17] S. Chen, G. Wang, J. Wen, and X. Wen, "Wave propagation and attenuation in plates with periodic arrays of shunted piezo-patches," Journal of Sound and Vibration, 2012.

[18] M. Collet, M. Ouisse, and M. Ichchou, "Structural energy flow optimization through adaptive shunted piezoelectric metacomposites," Journal of Intelligent Material Systems and Structures, vol. 23, no. 15, pp. 1661-1677, 2012.

[19] M. Collet, M. Ouisse, M. N. Ichchou, and R. Ohayon, "Semiactive optimization of $2 \mathrm{D}$ wave dispersion into shunted piezo-composite systems for controlling acoustic interaction," Smart Materials and Structures, vol. 21, no. 9, p. 094002, 2012. [Online]. Available: http://iopscience.iop.org/0964-1726/21/9/094002

[20] B. Beck, K. Cunefare, M. Ruzzene, and M. Collet, "Experimental analysis of a cantilever beam with a shunted piezoelectric periodic array," Journal of Intelligent Material Systems and Structures, vol. 22, pp. 1177-1187, 2011. 